\title{
Perfil antropométrico de idosos atendidos pela atenção primária de saúde do município de Caicó - Estudo piloto para nortear intervenções de equipes multiprofissionais em saúde
}

\author{
Anthropometric profille of elderly people assisted by primary health care in the city of Caicó - Pilot \\ study to guide interventions by multidisciplinary health teams \\ Perfil antropométrico de ancianos atendidos por atención primaria de salud en la ciudad de Caicó - \\ Estudio piloto para orientar intervenciones de equipos multidisciplinarios de salud
}

\section{Resumo}

Conhecer o perfil antropométrico de idosos torna-se importante na intervenção multiprofissional em saúde, contribuindo para a redução das taxas de morbimortalidade, controle e prevenção de doenças associadas à obesidade. Este estudo tem como objetivo descrever o perfil antropométrico de idosos atendidos pela atenção primária de saúde de Caicó. É um estudo piloto, caracterizado como individuado, observacional e transversal. Os dados foram coletados em Unidades Básicas de Saúde de Caicó de março a abril de 2020. Nesse estudo participaram 30 indivíduos com 60 anos ou mais, a maioria do sexo feminino. Dentre as variáveis antropométricas apresentadas pela amostra estão a estatura, peso corporal, circunferência da cintura e do quadril, índice de massa corporal e relação cintura-quadril. Os resultados desta pesquisa mostram a estatura variando entre 1,33 e $1,70 \mathrm{~m}$; o peso corporal entre 36 e $91,7 \mathrm{~kg}$; a circunferência da cintura entre 62 e $118 \mathrm{~cm}$; a circunferência do quadril entre 80 e $125 \mathrm{~cm}$; o índice de massa corporal demonstrou que $40 \%$ dos idosos são eutróficos, $40 \%$ possuem sobrepeso e $20 \%$ estão com baixo peso; quanto a relação cintura-quadril observou-se que no sexo feminino há predominância de um alto risco, enquanto no sexo masculino uma predominância de médio risco. Os achados apontam tendência ao sobrepeso, onde a maioria dos idosos estão acima dos parâmetros antropométricos recomendados pelos principais órgãos de saúde, e ainda, que esses dados poderão contribuir para o planejamento de estratégias que possam servir de norte para intervenções de equipes multiprofissionais em saúde que atuam na atenção primária de saúde.

Palavras-chave: População idosa; Atenção primária de saúde; Equipe multiprofissional; Unidade Básica de Saúde. 


\begin{abstract}
Knowing the anthropometric profile of the elderly becomes important in multiprofessional health intervention, contributing to the reduction of morbidity and mortality rates, control and prevention of diseases associated with obesity. This study aims to describe the anthropometric profile of elderly people assisted by primary health care in Caicó. It is a pilot study, characterized as individual, observational and cross-sectional. Data were collected in Basic Health Units in Caicó from March to April 2020. In this study, 30 individuals aged 60 years or more participated, most of them female. Among the anthropometric variables presented by the sample are height, body weight, waist and hip circumference, body mass index and waist-hip ratio. The results of this research show the height varying between 1.33 and $1.70 \mathrm{~m}$; body weight between 36 and $91.7 \mathrm{~kg}$; waist circumference between 62 and $118 \mathrm{~cm}$; hip circumference between 80 and $125 \mathrm{~cm}$; the body mass index showed that $40 \%$ of the elderly are eutrophic, $40 \%$ are overweight and $20 \%$ are underweight; as for the waist-hip ratio, it was observed that in females there is a predominance of high risk, while in males a predominance of medium risk. The findings point to a tendency towards overweight, where most elderly people are above the anthropometric parameters recommended by the main health agencies, and that these data may contribute to the planning of strategies that can serve as a guide for interventions by multidisciplinary health teams that work in primary health care.
\end{abstract}

Keywords: Elderly population; Primary health care; Multiprofessional team; Basic Health Unit.

\title{
Resumen
}

Conocer el perfil antropométrico del anciano cobra importancia en la intervención sanitaria multiprofesional, contribuyendo a la reducción de las tasas de morbilidad y mortalidad, control y prevención de enfermedades asociadas a la obesidad. Este estudio tiene como objetivo describir el perfil antropométrico de las personas mayores atendidas por la atención primaria de salud en Caicó. Se trata de un estudio piloto, caracterizado por ser individual, observacional y transversal. Los datos fueron recolectados en Unidades Básicas de Salud en Caicó de marzo a abril de 2020. En este estudio participaron 30 individuos de 60 años o más, la mayoría mujeres. Entre las variables antropométricas que presenta la muestra se encuentran la altura, el peso corporal, la circunferencia de cintura y cadera, el índice de masa corporal y la relación cintura-cadera. Los resultados de esta investigación muestran que la altura varía entre 1,33 y 1,70 m; peso corporal entre 36 y 91,7 kg; circunferencia de la cintura entre 62 y $118 \mathrm{~cm}$; circunferencia de la cadera entre 80 y $125 \mathrm{~cm}$; el índice de masa corporal mostró que el $40 \%$ de los ancianos son eutróficos, el $40 \%$ tienen sobrepeso y el $20 \%$ tienen bajo peso; En cuanto a la relación cintura-cadera, se observó que en las mujeres predomina el riesgo alto, mientras que en los hombres predomina el riesgo medio. Los hallazgos apuntan a una tendencia hacia el sobrepeso, donde la mayoría de las personas mayores se encuentran por encima de los parámetros antropométricos recomendados por las principales agencias de salud, y que estos datos pueden contribuir a la planificación de estrategias que puedan servir de guía para las intervenciones de los equipos de salud multidisciplinarios que trabajan en atención primaria de salud.

Palabras clave: Poblacion vieja; Primeros auxilios; Equipo multiprofesional; Unidad Básica de Salud.

\section{Introdução}

É habitual afirmar que a população brasileira está envelhecendo de maneira jamais observada. E esse processo de envelhecimento, que em alguns países da Europa demorou mais de 100 anos, o Brasil está alcançando em menos da metade do tempo como mostra a famosa pirâmide etária do Instituto Brasileiro de Geografia e Estatística (IBGE) que dispõem vem assumindo gradativamente uma geometria de pirâmide invertida (Gomes, 2021). Chegar à velhice se transformou em uma das maiores conquistas dos últimos séculos, no entanto, se tornou o grande desafio da atualidade (Veras \& Oliveira, 2018). De acordo com Lima e Delgado (2017) o envelhecimento pode ser conceituado como um conjunto de modificações morfológicas, fisiológicas, bioquímicas e psicológicas, que determinam a perda progressiva da capacidade de adaptação do indivíduo ao meio ambiente, sendo considerado um processo dinâmico e progressivo. Segundo o IBGE (2019), o Brasil é um país com muitos idosos, gerando assim grandes demandas para políticas públicas de saúde para atender esse público. Considera-se que ao passar dos 60 anos de idade a pessoa necessita de uma série de cuidados que poderá favorecer o prolongamento de vida com saúde, autonomia e disposição.

Atualmente, a Organização Panamericana de Saúde (2019) recomendou aos países da Região das Américas que fortaleçam seus sistemas de saúde e de proteção social para atenderem às demandas de cuidados de longa duração que, em quantidade, triplicarão nas próximas três décadas, passando de oito milhões a 27 ou a 30 milhões até 2050. No mesmo sentido, a Organização dos Estados Americanos (2015), em 15 de junho de 2015, já havia aprovado a Convenção Interamericana sobre 
a Proteção dos Direitos Humanos da Pessoa Idosa, por meio de um documento juridicamente vinculante. Um item especifico dispõe que os "Estados Partes devem adotar medidas para desenvolver um sistema integral de cuidados que leve em conta a perspectiva de gênero e o respeito à dignidade e integridade física e mental do idoso, para garantir-lhe o gozo efetivo de seus direitos humanos por meio de cuidados de longo prazo".

Cabe destacar o papel da Atenção Primária de Saúde (APS) e suas equipes multiprofissionais em saúde possuem significativa importância nesse processo, visto que, servem de base para o modelo de assistência e organização dos sistemas de saúde. A Estratégia Saúde da Família (ESF), por sua vez, configura-se como uma reorientação do modelo assistencial em saúde na APS que busca garantir a integralidade da assistência centrada na família e direcionada à comunidade. Pelos idosos representarem uma das parcelas mais vulneráveis da população, portanto, espera-se que os serviços oferecidos pelas equipes de multiprofissionais do Sistema Único de Saúde (SUS) sejam capazes de se organizar e ofertar serviços aos idosos considerando suas demandas específicas e busca pela redução das iniquidades em saúde (Brasil, 2012).

Partindo dessa premissa, o presente estudo teve como objetivo descrever o perfil antropométrico de idosos atendidos pela atenção primária de saúde do município de Caicó, auxiliando no planejamento e direcionamento de estratégias metodológicas e estruturais dos programas de saúde oferecidos a essa população.

\section{Metodologia}

O presente estudo é do tipo piloto, caracterizado como individuado, observacional, transversal e abordagem quantitativa. A pesquisa foi realizada em 3 unidades básicas de saúde (UBS's) do município de Caicó, Rio Grande do Norte (RN). Caicó é um município brasileiro pertencente ao estado do RN, localizado na zona central do estado, distante $282 \mathrm{~km}$ da capital de estado, Natal. A coleta dos dados somente nas 3 UBS's se deu em decorrência da epidemia do COVID-19 que dificultou, devido a eminência da pandemia do COVID-19 que culminou com a suspensão da coleta, que a pesquisa se estendesse para UBS's de outras regiões do município pesquisado. O recrutamento foi feito a partir de conversas com os agentes comunitários de saúde que estenderam o convite a população de suas áreas de atuação.

A população do estudo foi constituída por indivíduos com 60 anos ou mais, atendidos pela APS da zona urbana do município de Caicó. De acordo com o último IBGE (2020), o município de Caicó possui, aproximadamente, 7.716 pessoas com 60 anos ou mais, considerando a população urbana e rural (https://cidades.ibge.gov.br/). Além disso, sabe-se que o município apresenta 21 UBS's com 95\% de abrangência populacional.

A amostra foi restrita a 3 UBS's da zona leste do município de Caicó, sendo representativo apenas para um contexto restrito da zona leste do município de Caicó, porém, podendo ser reproduzido em outros contextos. Os critérios de elegibilidade foram: idosos com 60 anos ou mais, de ambos os sexos; comunitários cadastrados nas Unidades Básicas de Saúde da zona urbana do município de Caicó (RN); não estar temporária ou permanentemente acamado; não ser encontrado, durante o recrutamento, para avaliação após três tentativas; não conseguir concluir todas as etapas da coleta dos dados.

Os dados foram coletados no período de fevereiro a março de 2020, por uma equipe devidamente treinada para aplicar os instrumentos de coleta. Os participantes foram convidados a participar da pesquisa e assinar o Termo de Consentimento Livre Pós-esclarecido. As informações coletadas foram referentes a: características sociodemográficas, dados antropométricos, condições de saúde, prática de atividade física e razões motivacionais para prática de atividade física.

Quanto às características sociodemográficas, foram coletadas as seguintes informações: sexo, idade, estado civil, o exercício ou não de trabalho remunerado e grau de escolaridade. Foram tomadas medidas antropométricas da estatura, peso corporal, circunferências da cintura e do quadril. Na aferição da estatura, foi utilizado um estadiômetro transportável, da marca SANNY®, com $212 \mathrm{~cm}$ e precisão de $0,1 \mathrm{~cm}$, solicitando que os idosos tirassem os calçados e se colocassem de costas para o estadiômetro, em posição ereta e com os pés unidos (Charro, 2020). Na verificação do peso corporal, foi utilizada uma balança 
digital portátil da marca SPEEDO®, com capacidade de $150 \mathrm{Kg}$ e com precisão de 100g. Pediu-se que as mesmas retirassem os calçados e objetos, instruiu-se para que ficassem em pé sobre a plataforma da balança, com afastamento lateral dos pés, eretos e com olhar fixo para a frente (Charro, 2020). A medida da circunferência da cintura, utilizou-se uma fita métrica flexível com precisão de $1 \mathrm{~mm}$, da marca $S A N N Y ®$, e a medição foi realizada com o idoso em pé, circundando-se a fita na linha natural da cintura, na região mais estreita entre o tórax e o quadril, no ponto médio entre a última costela e a crista ilíaca (Charro, 2020). Na circunferência do quadril, utilizou-se uma fita métrica flexível com precisão de $1 \mathrm{~mm}$, da marca SANNY®, realizada o idoso em pé, pernas unidas, circundando-se a fita na linha natural do quadril, na região mais volumosa dos glúteos (Charro, 2020).

Utilizou-se, para a análise do Índice de Massa Corporal (IMC), calculado a partir da divisão da massa corporal em quilogramas pela estatura elevada ao quadrado $\left(\mathrm{kg} / \mathrm{m}^{2}\right)$ e os pontos de corte recomendados pelo Ministério da Saúde (Brasil, 2004) para avaliação da população idosa. Com base no IMC para idosos, foram considerados baixo-peso o IMC $\leq 22 \mathrm{~kg} / \mathrm{m}^{2}$, eutrófico $22>I M C>27 \mathrm{~kg} / \mathrm{m}^{2}$ e sobrepeso o IMC $\geq 27 \mathrm{~kg} / \mathrm{m}^{2}$ (Who, 2000).

A verificação do risco de desenvolvimento de complicações associadas à obesidade, através da relação das circunferências da cintura e do quadril (IRCQ), tomando por base os valores sugeridos por sexo pela Organização Mundial da Saúde (Who, 2008) que considera que, para mulheres, valores acima de 0,82 representam um risco aumentado, e para homens e acima de 0,94 . A condição de saúde foi avaliada a partir da autopercepção da saúde dos idosos. A autopercepção de saúde foi avaliada perguntando ao idoso, a partir de sua concepção subjetiva, como ele considera sua saúde, cuja resposta varia entre muito boa, boa, regular, ruim ou muito ruim.

Os dados foram tabulados e armazenados e processados no software estatístico Statistical Package for the Social Science versão 20.0 para Windows (SPSS 20.0). A análise estatística constou de análise descritiva (frequências, medidas de tendência central e medidas de dispersão) para caracterizar a amostra quanto às características sociodemográficas, às condições de saúde e os hábitos de vida. Em seguida, para uma melhor compreensão da motivação para a prática de atividade física, foi realizada uma análise bivariada (Teste t-student ou ANOVA) para comparação das médias dos escores brutos das dimensões em relação a variáveis independentes de condições de saúde.

O estudo foi aprovado pelo Comitê de Ética em Pesquisa da Faculdade de Ciências da Saúde (CEP FACISA/UFRN), conforme as determinações da Resolução n ${ }^{\circ}$ 466/12 do Conselho Nacional de Saúde (CNS), que define as diretrizes e normas regulamentadoras que regem a pesquisa envolvendo seres humanos, conforme as suas recomendações, tendo registro no CAAE: 24485819.7.0000.5568 e Número do Parecer: 3.737.785.

\section{Resultados e Discussão}

Ao todo este estudo pesquisou 30 idosos de 60 anos ou mais atendidos pelas mais diversas Unidades Básicas de Saúde da APS do município de Caicó no período de março a abril de 2020. A tabela I apresenta a amostra composta em sua maioria pelo sexo feminino (86,7\%), com média de idade de 71,6 $\pm 6,3$ anos, estado civil casado (36,7\%), escolaridade inferior ao Ensino Fundamental completo (63,3\%) e sem exercer trabalho remunerado (80\%). Quanto à condição e concepção de saúde os entrevistados responderam ter uma saúde excelente (3,3\%), muito boa (23,3\%), boa (20\%), regular (43,3\%) e ruim (10\%); e quanto aos hábitos de vida, a maioria dos indivíduos relataram não fumar $(83,3 \%)$ e não ser etilistas $(93,3 \%)$.

A tabela 1 apresenta a relação das variáveis antropométricas, características sociodemográficas, condições de saúde e hábitos de vida, IMC e IRCQ, onde observou-se que a estatura da amostra pesquisada variou entre $1,33 \mathrm{~m}$ e $1,70 \mathrm{~m}$; o peso corporal variou entre $36 \mathrm{~kg}$ e $91,7 \mathrm{~kg}$; a circunferência da cintura variou entre $62 \mathrm{~cm}$ e $118 \mathrm{~cm}$; a circunferência do quadril esteve entre $80 \mathrm{~cm}$ e $125 \mathrm{~cm}$. De acordo com o IMC, a amostra apresentou resultados onde, $40 \%$ dos idosos foram considerados eutróficos, $40 \%$ com sobrepeso e $20 \%$ com baixo peso; percebe-se ainda que em relação desenvolvimento de complicações 
associadas à obesidade (IRCQ), os valores do IRCQ para o sexo feminino há predominância de um IRCQ de Alto Risco, enquanto no sexo Masculino uma predominância de um IRCQ de Médio Risco. Ao tocante à pratica de atividade física, a maior parte dos entrevistados relataram praticar atividade regularmente (73,3\%); sendo que a atividade física de intensidade leve foi a mais relatada $(43,3 \%)$.

Tabela 1: Caracterização da amostra quanto a: características sociodemográficas, condições de saúde e hábitos de vida. Caicó (RN). 2020.

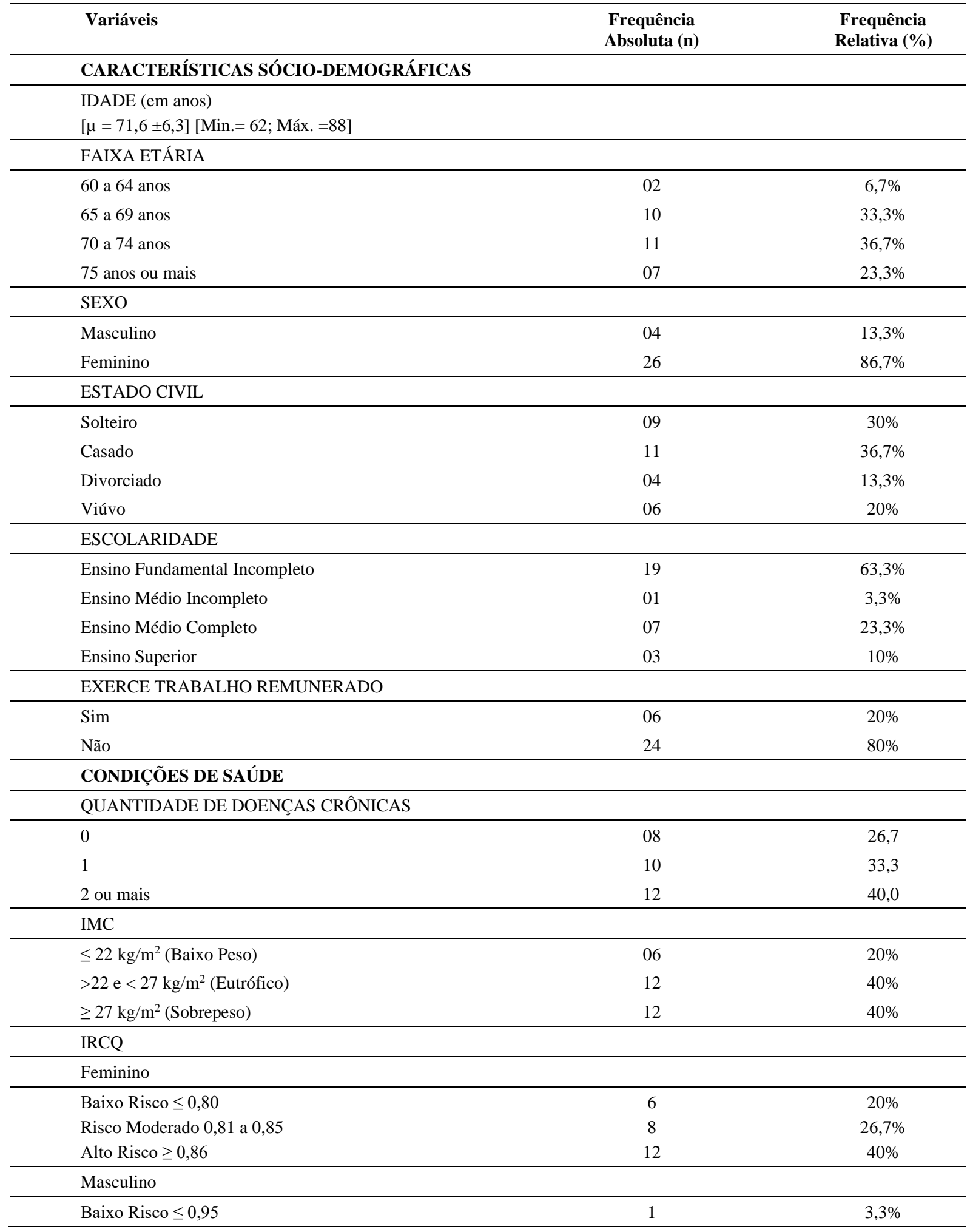




\begin{tabular}{lcc}
\hline Risco Moderado 0,96 a 1,0 & 2 & $6,7 \%$ \\
Alto Risco $\geq 1,0$ & 1 & $3,3 \%$ \\
\hline PERCEPÇÃO DE SAÚdE & 08 & $26,7 \%$ \\
\hline Excelente/ Muito Boa & 06 & $20 \%$ \\
Boa & 13 & $43,3 \%$ \\
Regular & 03 & $10 \%$ \\
Ruim & & $16,7 \%$ \\
\hline HÁBITOS DE VIDA & $83,3 \%$ \\
\hline USO DE TABACO & 05 & $6,7 \%$ \\
\hline Fumante & 25 & $93,3 \%$ \\
Não fumante & & \\
\hline CONSUMO DE ÁLCOOL & 02 & $73,3 \%$ \\
\hline Etilista & 28 & $26,7 \%$ \\
Não etilista & & $43,3 \%$ \\
\hline PRATICA ATIVIDADE FÍSICA & 22 & $40 \%$ \\
\hline Sim & 08 & $16,7 \%$ \\
\hline Não & & 13 \\
\hline TIPO DE ATIVIDADE FÍSICA & 12 & 05 \\
\hline Lenta / leve & & \\
Moderada & & \\
Rápida / vigorosa & & \\
\hline
\end{tabular}

Fonte: Autores.

Este estudo teve como objetivo descrever o perfil antropométrico de idosos com 60 anos ou mais atendidos pela atenção primária a saúde do município de Caicó, assim como as características sociodemográficas, condições de saúde e hábitos de vida dos idosos.

No presente estudo, foi possível obter uma amostra de 30 indivíduos, com média de idade de 71,3 anos \pm 6,3; sendo $86,7 \%$ do sexo feminino e 13,3\% masculino. A maior participação de idosos do sexo feminino pode estar relacionada a feminização do envelhecimento e à maior capacidade de autocuidado por parte das mulheres. Corroboram com este resultado os estudos de Silva e Kraeski (2010), Eiras et al., (2010), Massetto (2011) e Schwingel (2011) que também apresentaram a predominância de mulheres envolvidas em programas de atividades físicas. Por outro lado, López-Benavente et al., (2018) em estudo realizado com idosas, afirmou que a as mulheres tendem a apresentar fatores de desmotivação para a prática de exercício, pois são sobrecarregadas com tarefas domésticas, influenciando uma precária percepção de saúde e pouco tempo livre.

A maioria dos idosos do presente estudo relatou ter união estável, escolaridade inferior ao ensino fundamental completo, não exercer atualmente trabalhado remunerado, ser não tabagista e não etilista. Estudos envolvendo participantes de programas de atividade física oferecidas por UBS's apresentou características sociodemográficas semelhantes (Carvalho et al., 2016; Lovato et al., 2015; Silva et al., 2011).

As alterações físicas (peso e estatura) resultantes do processo de envelhecimento podem influenciar nas dimensões corporais, que quando muito alteradas, interferem na manutenção da saúde, influenciam negativamente na qualidade de vida e na morbimortalidade, por essa razão, a avaliação do estado nutricional na população idosa é muito importante. Destaca-se que, nessa população, alterações nutricionais, marcadas pelo baixo-peso, são tão prejudiciais quanto pelo excesso de peso. (Sass \& Marcon, 2015).

Atualmente, o sobrepeso e a obesidade constituem problema de saúde pública e são prevalentes também na população idosa, o que tem despertado a atenção dos profissionais de saúde quanto à necessidade de planejamento de estratégias que 
visem conscientizar a população em geral, quanto aos riscos que essa alteração traz para a saúde em todos os ciclos da vida (Da Silva, Souza, Petroski, Silva, 2011).

Sabe-se que muitos motivos podem influenciar a composição corporal, dentre eles o gênero, o nível socioeconômico, a escolaridade (Krug, 2012), a autopercepção de saúde (Souza \& Vendrúsculo, 2010), o estado civil, a obesidade, o tabagismo e o álcool (Hirvensalo \& Lintunen, 2011), as condições ambientais, de transporte e de segurança (Cassou et al., 2011), além da imagem negativa do idoso perante a sociedade (Moschny et al., 2011).

Observou-se nesse estudo que a circunferência da cintura da população estudada esteve compreendida entre $62 \mathrm{~cm} \mathrm{e}$ $118 \mathrm{~cm}$, onde sabe-se que medidas altas da circunferência da cintura estão associadas a maior risco para doenças cardiovasculares e preditores confiáveis de excesso de gordura visceral, como obesidade geral. A obesidade central aferida pela circunferência da cintura tem sido proposta como um dos melhores preditores antropométricos de gordura visceral e risco de doenças metabólicas, tendo maior associação com mortalidade e mais fortemente correlacionada com fatores de risco (Silveira et. al., 2020).

Resultados encontrados nesse estudo evidenciaram sobrepeso e/ou obesidade em $40 \%$ dos participantes, o que confirmam a prevalência de obesidade entre idosos, principalmente, do sexo feminino, uma realidade observada em outros estudos (Sass \& Marcon, 2015; Silveira, Kac, Barbosa, 2009). Segundo Malta et al. (2014), a prevalência do excesso de peso aumentou, em todo território nacional, significativamente, em ambos sexos, em todas as faixas etárias e em todos os níveis de escolaridade. O estudo de Silva et al. (2018), realizado com 35.214.802 idosos de 60 anos ou mais de idade, também evidenciou a tendência ao aumento da prevalência de sobrepeso e de obesidade nas cinco regiões do país, reforçando a ocorrência da transição nutricional no Brasil. Já o baixo-peso correspondeu somente a $20 \%$ da população deste estudo.

$\mathrm{O}$ estudo trouxe uma maior proporção de idosos com relação cintura-quadril classificado em risco moderado ou elevado, sendo o sexo feminino há predominância de um alto risco, enquanto no sexo masculino uma predominância de médio risco, tais achados, reforçam ao risco aumentado do aparecimento de doenças cardiometabólicas. Corroboram que esse resultado o estudo de Moreira et. al. (2009) utilizando a relação cintura-quadril, trouxe dados em quem homens apresentaram classificação de "risco moderado" e em mulheres "risco alto" para o desenvolvimento de complicações metabólicas, respectivamente. Sabe-se que há diferenças no padrão do comportamento adiposo corporal entre os sexos dos indivíduos idosos, com maior tendência de acúmulo de gordura na região central das mulheres. Já nos homens, a gordura tende a concentrar em maior quantidade nas regiões periféricas corporais. Essas disparidades apontam que tanto a CC quanto o IMC são relevantes e complementares na análise da obesidade, sendo a primeira mais eficiente para predizer o risco às doenças endócrinas e metabólicas, e o segundo para identificar as reservas energéticas e estimar a gordura corporal total. O estudo de Araújo et. al., (2018) denota a inter-relação no uso dos dois indicadores antropométricos envolve o fato de a obesidade abdominal ter mostrado associações significativas somente para o sexo feminino. Sabe-se que há diferenças no padrão do comportamento adiposo corporal entre os sexos dos indivíduos idosos, com maior tendência de acúmulo de gordura na região central das mulheres. Já nos homens, a gordura tende a concentrar em maior quantidade nas regiões periféricas.

Cabe ressaltar que o presente estudo apresenta limitações. O tempo de coleta de dados e a quantidade de idosos pesquisados devido a eminência da pandemia do COVID-19 que culminou com a suspensão da coleta. O tamanho da amostra foi um fator limitante com importante influência no poder estatístico. A realização do estudo em uma região específica, com amostra por conveniência, inviabilizou que os resultados obtidos sejam extrapolados para outras populações. Análises comparativas mais elaboradas entre os sexos não foram possíveis de serem realizadas devido ao tamanho amostral e a quantidade de participantes do sexo masculino, mas consiste em uma interessante estratégia de análise, considerando-se diferentes parâmetros antropométricos para homens e mulheres. Estudos futuros, com amostras maiores e delineamentos de pesquisa mais sofisticados, são fundamentais para a obtenção de resultados mais robustos, sugere-se que mais estudos com 
amostras ainda mais expressivas sejam realizados na intenção de compreender as razões motivacionais para prática de atividades físicas em idosos atendidos pela APS, pois entende-se a importância de programas de atividades físicas na vida desta população, pois se trata de uma forma de mantê-los em vida social ativa e saudável tendo em vista as projeções para o aumento de idosos mundialmente, já vividas atualmente.

\section{Conclusão}

A realização desta pesquisa possibilitou, além do perfil antropométrico, caracterizar o perfil sociodemográfico e de saúde de idosos atendidos pela APS da zona leste do município de Caicó (RN). Onde a maioria dos participantes foi do sexo feminino, chamando a atenção para a necessidade de um maior envolvimento de idosos do sexo masculino nas ações desenvolvidas pelas equipes multiprofissionais de saúde das UBS's, tornando-se importante o direcionamento de atividades e cuidados específicos para os idosos. Os resultados mostraram diferenças nos valores médios das variáveis antropométricas entre os sexos, com tendência ao sobrepeso e risco aumentado de aparecimento de doenças cardiometabólicas. A alta prevalência de obesidade na população em estudo, verificada por meio do índice de massa corporal, relação cintura-quadril indica a necessidade de controle e acompanhamento do peso, assim como o desenvolvimento de ações que visem estimular a adesão de hábitos saudáveis de vida. Os achados deste estudo contribuem na elaboração de estratégias por parte das equipes multiprofissionais em saúde para que atendam não só às expectativas deste público, como também auxiliem na manutenção, em longo prazo, desses indivíduos de forma a adotarem um estilo de vida ativo e saudável. Sugere-se a inserção e participação de profissionais e acadêmicos dos diversos cursos da área de saúde nas orientações gerais de prevenção, autocuidado e promoção da saúde, devido muitas vezes à carência de uma equipe multidisciplinar como visão holística para atender as necessidades individuais e coletivas desses idosos, assim como, a realização estudos populacionais sobre a temática para melhor conhecer o perfil da população idosa, e ainda, que os estudos futuros, relacionados a essa temática, possam trazer uma amostra com um número maior de indivíduos afim de trazer dados ainda concretos.

\section{Referências}

Araújo, C. A. H., Giehl, M. W. C., Danielewicz, A. L., Araújo, P. G., D’Orsi, E., \& Boing, A. F. (2018). Ambiente construído, renda contextual e obesidade em idosos: evidências de um estudo de base populacional. Cad. Saúde Pública, 34(5):e00060217.

Cassou, A. C. N. et al. (2011). Barriers to physical activity among brazilian elderly women from different socioeconomic status: a focus-group study. Journal of physical activity and health. 8, $126-132$.

Carvalho, F. F. B., \& Nogueira, J. A. D. (2016). Práticas corporais e atividades físicas na perspectiva da promoção da saúde na atenção básica. Ciência \& Saúde Coletiva. 21(6):1829-1838.

Charro, M. A., Figueira Júnior, A., \& Allegretti, J. G. (2020). Tratado de musculação. Ed. Phorte.

Da Silva, V. S., Souza, I., Petroski, E. L., \& Silva, D. A. S. (2011). Prevalência e fatores associados ao excesso de peso em idosos brasileiros. Rev Bras Ativ Fís Saúde, 16(4):289-94.

Eiras, S. B., Silva, W. H. A., Souza, D. L., \& Vendruscolo, R. (2010). Fatores de adesão e manutenção da prática de atividade física por parte de idosos. Revista brasileira de ciências do esporte. 31(2):75-89.

Gomes, I. K. (2021). Avaliação e Prescrição Clínica de Exercício Físico para Grupos Especiais. Lura Editorial.

Hirvensalo, M., \& Lintunen, T. (2011). Life-course perspective for physical activity and sports participation. European Revew of Aging and Physical Activity. v. 8 , p. $13-22$.

IBGE. (2020). Estimativas populacionais para municípios brasileiros. http://www.ibge.gov.br.

IBGE. (2019). População idosa. http://www.ibge.gov.br.2019.

Knuth, A. G., Bielemann, R. M., Silva, S. G., Borges, T. T., \& Del Duca, G. F. (2009). Conhecimento de adultos sobre o papel da atividade física na prevenção e tratamento de diabetes e hipertensão: estudo de base populacional no sul do Brasil. Caderno de saúde pública. 25(3):513-20.

Krug, R. R. (2012). Idosas longevas inativas fisicamente: percepção das barreiras e facilitadores para a prática da atividade física. [Dissertação mestrado em ciências do movimento humano]. Florianópolis: UESC. 
Lima, A. P., Delgado, E. I. (2017). A melhor idade do Brasil: aspectos biopsicossociais decorrentes do processo de envelhecimento. ACTA Brasileira do Movimento Humano.

López-benavente, Y., Arnau-sánchez, J., Ros-sánchez, T., Lidón-cerezuela, M. B., Serrano-noguera, A., \& Medina-abellán, M. D. (2018). Difficulties and motivations for physical exercise in women older than 65 years. A qualitative study. Revista Latino-americana de Enfermagem. 26, 1-10.

Lovato, S. S., Loch, M. R, Gonzávez, A. D., \& Lopes, M. L. S. (2015). Assiduidade a programas de atividade física oferecidas por unidades básicas de saúde: o discurso de participantes muito e pouco assíduos. Revista Brasileira de Atividade Física e Saúde. 20(2):184-192.

Malta, D. C., Andrade, S. C., Claro, R. M., Bernal, R. T. I., \& Monteiro, R. C. A. (2014). Evolução anual da prevalência de excesso de peso e obesidade em adultos nas capitais dos 26 estados brasileiros e no distrito federal entre 2006 e 2012. Revista Brasileira de Epidemiologia. 2014 ; $267-76$.

Massetto, S. T. (2011). Manutenção da prática de atividades aquáticas: tempo de prática e alterações dos principais motivos, na percepção dos idosos. Revista Mackenzie de Educação Física e Esporte. 10, 76-88.

Matsudo, S. M. (2009). Envelhecimento, atividade física e saúde. Bol. Inst. Saúde. 47.

Moreira, A. J., Nicastro, H., Cordeiro, R. C. C., Coimbra, P., \& Frangella, V. S. (2009). Composição corporal de idosos segundo a antropometria. Rev. Bras. Geriatr. Gerontol, 12(2):201-213.

Moschny, A., Platen, P., Klaassen-mielke, R., Trampisch, U., \& Hinrichs, T. (2011). Barriers to physical activity in older adults in germany: a cross- -sectional study. Int J Behav Nutr Phys Act. 8(1):121.

OEA. (2015). Convenção Interamericana sobre a Proteção dos Direitos Humanos dos Idosos. OEA.

OPAS. (2019). Plano de ação sobre a Saúde dos Idosos, incluindo o envelhecimento ativo e saudável: Relatório Final. OPAS.

Sass, A., \& Marcon, S. S. (2015) Comparação de medidas antropométricas de idosos residentes em área urbana no sul do Brasil, segundo sexo e faixa etária. Rev. Bras. Geriatr. Gerontol., 18(2):361-372.

Schwingel, V. (2011). Aderência de idosos no projeto de hidroginástica do projetar. 2011. 57f. [Monografia Bacharelado em Educação Física]. UFRS.

Silva, H. O., Carvalho, M. J. A. D., Lima, F. E. L. L., \& Rodrigues, L. V. (2011). Perfil epidemiológico de idosos frequentadores de grupos de convivência no município de Iguatu (CE). Revista Brasileira de Geriatria e Gerontologia. 14(1): 123-33.

Silva, L. C., \& Kraeski, D. (2010). Motivos de adesão e permanência em programas de atividade física por idosos. 2006. 10f. [Artigo Licenciatura em Educação Física - Centro de Ciências da Saúde e do Esporte]. UESC.

Silva, V. S., Souza, I., Silva, D. A. S., Barbosa, A. R., \& Fonseca, M. J. M. (2018). Evolução e associação do IMC entre variáveis sociodemográficas e de condições de vida em idosos do Brasil: 2002/03-2008/09. Ciência \& Saúde Coletiva. 23(3):891-901.

Siveira, E. A., Pagotto, V., Barbosa, L. S., Oliveira, C., Pena, G. G., \& Velasque-Melendez, G. (2020). Acurácia de pontos de corte de IMC e circunferência da cintura para a predição de obesidade em idosos. Ciência \& Saúde Coletiva, 25(3):1073-1082.

Silveira, E. A, Kac, G., \& Barbosa, L. S. (2009). Prevalência e fatores associados à obesidade em idosos residentes em Pelotas, Rio Grande do Sul, Brasil: classificação da obesidade segundo dois pontos de corte do índice de massa corporal. Cad Saúde Pública, 25(7):1569-77.

Veras, R. P. \& Oliveira, M. (2018). Envelhecer no Brasil: a construção de um modelo de cuidado. Ciência \& Saúde Coletiva. 23(6):1929-1936.

WHO. (2011). Global recommendations on physical activity for Health.

WHO. (2008). Waist circumference and waist-hip ratio. Report of a WHO expert consultation.

WHO. (2000). Obesity: preventing and managing the global epidemic. Report of a WHO Consultation. World Health Organization. 